\title{
INTOXICAÇÃO EXPERIMENTAL POR NARASINA EM OVINOS ${ }^{1}$
}

\author{
Flademir Wouters ${ }^{2}$, Angelica T. B. Wouters ${ }^{2}$ e Claudio S.L. Barros ${ }^{3}$
}

\begin{abstract}
Wouters F., Wouters A.T.B. \& Barros C.S.L. 1997. [Experimental narasin poisoning in sheep.] Intoxicação experimental por narasina em ovinos. Pesquisa Veterinária Brasileira 17(3/4):89-95. Depto Patologia, Universidade Federal de Santa Maria, RS 97119-900, Brazil.

Experimental poisoning by narasin was induced in 13 sheep through oral administration of the drug. Six sheep died, three were killed in extremis and four recovered. The onset of clinical signs was 2 hours to 7 days after the administration of the drug, and the clinical course lasted from 7 hours to 14 days. Initially there were laborious breathing, tachypnea, fever and ruminal atony. These signs were followed by incoordinated gait, stiffness, reluctance to move and changes in frequency and intensity of cardiac sounds. Grunting, grinding of teeth, myoglobinuria, and sternal and lateral recumbency were also observed. One sheep had sudden death. Necropsy findings were observed in seven of the sheep that died and consisted of pale areas in the skeletal muscles and myocardium. More frequently affected muscles were masseter, semitendinosus, sternocephalicus, brachiocephalicus, extrinsec muscles of the tongue, pectoral, supraspinatus and teres major. Hydropericardium, edema of the lungs and the wall of the gall bladder were also observed. Ocasionally the cut surface of the lymph nodes was reddened and moist. In one sheep there was edema surrounding the esophagus and within the cervical muscles; in another one there was a bolus of partially chewed food in the oral cavity. Histologically, the pale areas in the skeletal muscles and myocardium corresponded to multifocal or focal extensive degeneration and necrosis of myofibers; these lesions were sometimes associated with processes of regeneration in skeletal muscles and reparation in the myocardium. The histological muscle lesions were more pronounced in the tongue and muscles of the limbs.
\end{abstract}

INDEX TERMS: Narasin, ionophore antibiotics, sheep, pathology, toxicosis.

SINOPSE.- Intoxicação pelo antibiótico ionóforo narasina foi induzida experimentalmente em 13 ovinos; desses, seis morreram espontaneamente, três foram sacrificados in extremis e quatro se recuperaram. $\mathrm{O}$ início dos sinais clínicos ocorria entre 2 horas e 7 dias após a administração da droga e persistiam por 7 horas a 14 dias. Inicialmente havia respiração ofegante e entrecortada, taquipnéia, febre, redução temporária do apetite ou anorexia e atonia ruminal seguidos de alterações no andar, como arrastar das pinças, apoio sobre os

\footnotetext{
${ }^{1}$ Aceito para publicação em 25 de abril de 1997.

Parte da tese de mestrado do primeiro autor. Defendida no Curso de PósGraduação (CPG) em Medicina Veterinária, área de concentração em patologia, Universidade Federal de Santa Maria (UFSM), em 29 de janeiro de 1997. Trabalho financiado pelo CNPq, projeto 530198/93-2.

${ }^{2}$ CPG em Medicina Veterinária, área de concentração em patologia, Centro de Ciências Rurais, UFSM, 97119-900 Santa Maria, Rio Grande do Sul.

${ }^{3}$ Departamento de Patologia, UFSM. Bolsista do CNPq (350938/91-1).
}

boletos dos membros posteriores, incoordenação, rigidez, relutância em movimentar-se e alteração na frequência e intensidade dos sons cardíacos. Ocorriam também gemidos, ranger de dentes, urina acastanhada e decúbito esternal ou lateral. Um animal apresentou morte súbita. Alterações macroscópicas foram observadas em sete dos ovinos necropsiados e consistiam de áreas pálidas nos músculos esqueléticos e no miocárdio. Os músculos mais frequentemente atingidos foram masseter, semitendíneo, esternocefálico, braquicefálico, extrínsecos da língua, peitoral, supra-espinhal e redondo maior. Havia hidropericárdio, edema e congestão pulmonares, fígado pálido, edema da parede da vesícula biliar, linfonodos ocasionalmente avermelhados e suculentos ao corte, edema periesofágico e entre os feixes musculares da região cervical e acúmulo de alimento semimastigado acumulado na cavidade oral. Histologicamente as áreas pálidas dos músculos esqueléticos e do miocárdio correspondiam a lesões degenerativo-necróticas multifocais a focalmente extensas associadas ou não a processos 
regenerativos, nos músculos esqueléticos, e reparativos, no miocárdio. As lesões histológicas foram mais acentuadas nos músculos da língua e dos membros.

TERMOS DE INDEXAÇÃO: Narasina, antibióticos ionóforos, ovinos, patologia, intoxicação.

\section{INTRODUÇÃO}

Antibióticos ionóforos são substâncias que formam complexos químicos bipolares com cátions, facilitando o transporte iônico através de membranas biológicas. Alteram assim o equilíbrio iônico intracelular, resultando em tumefação celular aguda (Van Vleet et al. 1983). Têm sido usadas em medicina veterinária principalmente como coccidiostáticos para aves e bovinos e como promotores de crescimento para ruminantes, embora existam vários outros usos terapêuticos (Novilla 1992). Essas drogas são consideradas relativamente seguras se usadas nas espécies-alvo e dentro das dosagens recomendadas pelo fabricante. No entanto, seu uso inapropriado, acidental ou intencional,resulta em intoxicações de ovinos (Nation et al. 1982, Bourque et al. 1986), bovinos (Collins \& McCrea 1978, Geor \& Robinson 1985), equinos (Rollinson et al. 1987), suínos (Van Halderen et al. 1993, Miskimins \& Neiger 1996), cães (Karsai et al. 1990), coelhos (Salles et al. 1994) e aves (Behr et al. 1988). A intoxicação por antibióticos ionóforos caracteriza-se por miopatia e cardiomiopatia degenerativas. Nos últimos anos houve um acentuado aumento no uso terapêutico de antibióticos ionóforos em medicina veterinária, o que aumenta os riscos de intoxicação nos animais.

Esse trabalho tem como objetivo determinar o quadro clínico e o anatomopatológico da intoxicação por antibióticos ionóforos em ovinos.

\section{MATERIAL E MÉTODOS}

Quatorze ovinos sem raça definida, de ambos os sexos, com idades entre 1 e 2 anos foram divididos em sete lotes de dois ovinos e mantidos em baias de alvenaria. Os animais recebiam água fresca e feno de alfafa à vontade. Um composto contendo $10 \%$ de narasina ${ }^{4}$ diluído em água foi administrado oralmente a 13 ovinos (Quadro 1). Um ovino controle foi mantido durante todo o experimento sob as mesmas condições dos outros, não tendo recebido a droga.

Todos os ovinos eram submetidos a exame clínico pela manhã e no final da tarde. Diariamente eram retirados das baias e movimentados. Os animais que morreram espontaneamente ou foram sacrificados, incluindo o controle, foram necropsiados. Fragmentos de vários músculos esqueléticos (quadríceps femoral, semitendíneo, longissimus dorsi, diafragma, supra-espinhal, ocular e abdominal), miocárdio, língua, encéfalo, pulmão, fígado, esôfago, rúmen, abomaso, intestino delgado, intestino grosso, linfonodos mesentérico e pré-escapular foram colhidos e processados rotineiramente para histopatologia. Cortes selecionados de músculo esquelético e de miocárdio foram corados pelo tricrômico de Masson modificado por Goldner.

As alterações histológicas dos músculos esqueléticos e do miocárdio foram classificadas em degenerativo-necróticas e

\footnotetext{
${ }^{4}$ Monteban 100. Nutris Tecnologia e Sistema de Nutrição Ltda., BR 116, Km 73,5, Trevo Distrito Industrial, Quatro Barras, Paraná 83420-000.
}

Quadro 1. Delineamento experimental da intoxicação experimental por narasina em ovinos

\begin{tabular}{cccccc}
\hline Ovino & $\begin{array}{c}\text { Peso } \\
(\mathrm{kg})\end{array}$ & $\begin{array}{c}\text { Idade } \\
\text { (anos) }\end{array}$ & $\begin{array}{c}\text { Número de } \\
\text { administrações }\end{array}$ & $\begin{array}{c}\text { Administração } \\
\text { diária }(\mathrm{mg} / \mathrm{kg})\end{array}$ & $\begin{array}{c}\text { Total adminis- } \\
\text { trado(mg/kg) }\end{array}$ \\
\hline 01 & 27,7 & 2 & 1 & 30 & 30 \\
02 & 21,2 & 2 & 2 & 15 & 30 \\
03 & 26,0 & 1 & 1 & 15 & 15 \\
04 & 31,8 & 1 & 1 & 7 & 7 \\
05 & 34,0 & 1 & 1 & 4 & 4 \\
06 & 30,4 & 2 & 1 & 4 & 4 \\
07 & 35,7 & 1 & 1 & 2 & 2 \\
08 & 30,0 & 1 & 1 & 2 & 2 \\
09 & 30,0 & 1 & 14 & 1 & 12 \\
11 & 25,0 & 1 & 3 & 4 & 7 \\
12 & 24,8 & 1 & 3 & 4 & 7 \\
$13^{\text {a }}$ & 20,0 & 1 & 1 & 7 & 7 \\
14 & 20,0 & 1 & 1 & & \\
\end{tabular}

a'Os ovinos 13 e 14 eram fêmeas, os demais eram machos castrados. Um ovino (10) de 1 ano de idade, com $35 \mathrm{~kg}$, foi usado como controle, não tendo recebido a droga.

reparativas. Nas primeiras, foram incluídas vacuolização do sarcoplasma, necrose segmentar hialina e necrose segmentar flocular da miofibra. Nas alterações reparativas foram incluídas reações inflamatórias (incluindo fibrose) e regenerativas das miofibras.

\section{Sinais clínicos}

\section{RESULTADOS}

O tempo decorrido entre a administração de narasina e o aparecimento dos sinais clínicos, a duração do curso clínico e o desfecho da intoxicação constam do Quadro 2. O ovino 10 (controle) foi sacrificado logo após o término do experimento sem ter apresentado quaisquer sinais clínicos. Na maioria dos casos, os sinais clínicos iniciavam por respiração entrecortada (ovinos 1-5, 7, 11 e 13) e ofegante (ovinos 1-3, 5,11 e 13), e taquipnéia (ovinos 3 e 8); houve elevação da temperatura nos ovinos 1-3, 5 e 11. A intensidade dos sinais respiratórios diminuiu cerca de 18-30 horas após a administração da droga, mesmo nos ovinos que morreram. Anorexia (ovinos 1-3, 7 e 12) ou redução temporária do apetite (ovinos 4-6 e 8) foram sinais clínicos constantes. Dois ovinos (5 e 13) apresentaram mastigação vagarosa associada à salivação abundante com grandes pausas entre os movimentos mastigatórios e demora na deglutição. Um deles (ovino 13) apresentou dificuldade acentuada de apreensão do feno, deixando cair parte do feixe apreendido. As porções do feno que conseguia reter permaneciam por longo tempo parcialmente fora da boca (Fig. 1). Atonia ruminal foi observada em vários ovinos (1, 2, 12 e 13). Um desses animais (ovino 1) apresentou timpanismo discreto. Fezes pastosas foram observadas em um ovino (11).

Muitos animais levantavam somente quando forçados e mostravam relutância em caminhar (ovinos 1-3, 5 e 11). Esse sinal foi acentuado nos ovinos 5 e 11. Quando forçados a caminhar, os animais arrastavam as pinças dos membros posteriores (ovinos 1, 2, 5, 6, 9 e 13), tinham andar incoordenado (ovinos 1, 3, 5 e 11), rígido (ovinos $2,3,5,9$ e 11) e caminha- 

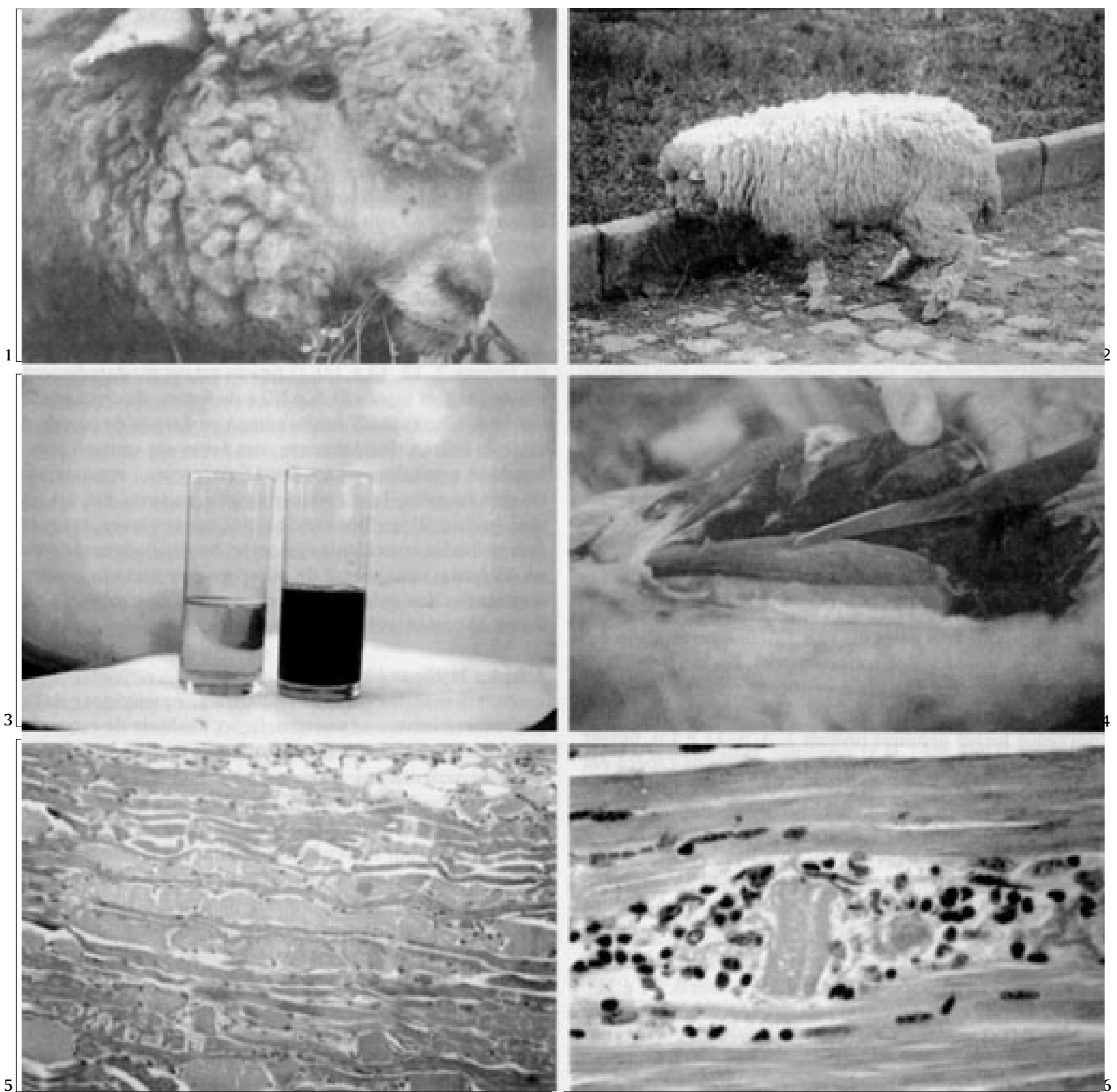

Fig. 1. Dificuldade de apreensão do feno. Esse animal apresentava mastigação lenta, ficando porções do feno apreendido por longo tempo fora da boca. Intoxicação experimental por narasina (ovino 13).

Fig. 3. Mioglobinúria, na intoxicação experimental por narasina. A urina do frasco à direita é do ovino 5 . Urina de um ovino normal à esquerda para comparação.

Fig. 5. Várias fibras do músculo abdominal mostram necrose hialina segmentar, na intoxicação experimental por narasina. Fibras normais ocorrem intercaladas entre as fibras necróticas (ovino 13$)$. HE, obj. 6,3

Fig. 2. Membros posteriores afastados e flexionados no jarrete, na intoxicação experimental por narasina (ovino 13).

Fig. 4. O músculo semitendíneo (ponta da faca) está difusamente pálido (branco amarelado) evidenciando miopatia degenerativa, na intoxicação experimental por narasina (ovino 5).

Fig. 6. O segmento necrótico de miofibra do músculo vasto lateral está invadido por células satélites e células inflamatórias, principalmente macrófagos, na intoxicação experimental por narasina; alguns poucos neutrófilos são também observados (ovino 13). HE, obj. 16. 
Quadro 2. Início, duração dos sinais clínicos após administração de narasina e desfecho da intoxicação experimental em ovinos

\begin{tabular}{cccl}
\hline Ovino & $\begin{array}{c}\text { Início dos sinais clínicos } \\
\text { após a primeira } \\
\text { administração }\end{array}$ & $\begin{array}{c}\text { Duração do curso } \\
\text { clínico }\end{array}$ & Desfecho \\
\hline 01 & $6 \mathrm{~h}$ & $40 \mathrm{~h}$ & Morreu \\
02 & $6 \mathrm{~h}$ & $24 \mathrm{~h}$ & Morreu \\
03 & $2 \mathrm{~h}$ & $7 \mathrm{~h}$ & Morreu \\
04 & $23 \mathrm{~h}$ & $3 \mathrm{~d}, 3 \mathrm{~h}$ & Morreu \\
05 & $2 \mathrm{~h} 30 \mathrm{~min}$ & 3 dias & Sacrificado \\
06 & $12 \mathrm{~h}^{\mathrm{a}}$ & 14 dias & Recuperou-se \\
07 & 5 & $20 \mathrm{~h}$ & Recuperou-se \\
08 & $3 \mathrm{~h}^{\mathrm{a}}$ & $20 \mathrm{~h}$ & Recuperou-se \\
09 & $7 \mathrm{dias}$ & 10 dias & Recuperou-se \\
11 & $2 \mathrm{~h}$ & 4 dias & Sacrificado \\
12 & $48 \mathrm{~h}$ & 4 dias & Morreu \\
13 & $2 \mathrm{~h}$ & 9 dias & Sacrificado \\
14 & - & - & Morreu \\
\hline
\end{tabular}

a Tempo aproximado.

vam sobre os boletos ( ovinos 2 e 3) ou com os membros afastados lateralmente (ovino 1 e 13) e flexionados no jarrete (Fig. 2).

Os ovinos 1, 4, 5-7,11,12 e 13 apresentaram arritmia cardíaca, sendo que três deles $(5,12$ e 13) tinham também aumento da intensidade dos sons cardíacos. Em um ovino (4) a arritmia cardíaca foi discreta e verificada somente cerca de uma hora antes da morte, que foi repentina. Outros sinais observados foram gemidos (ovinos $1,3,5,11$ e 13), ranger de dentes (1, 5 e13), apatia (1,5 e 12) e apoio da cabeça em objetos como parede da baia e cocho (ovinos 5 e 13), ocasionalmente mantendo parte do focinho dentro da água (ovino 13). Alguns animais pareciam, por vezes, inquietos, deitando-se e levantando-se repetidas vezes (3 e 11). Com o agravamento do quadro clínico, um animal permaneceu com a cabeça inclinada para o lado esquerdo (13). Urina escura, acastanhada (Fig. 3), foi observada nos ovinos 5 e 11. Em uma fase mais avançada da doença, alguns animais permaneciam em decúbito esternal (2 e 12) ou lateral (ovinos 5, 11 e 12), com o pescoço torcido, recostando a cabeça no flanco (2). Um animal (14) apresentou morte súbita três dias após a administração de narasina.

\section{Achados de necropsia}

As principais alterações macroscópicas dos ovinos intoxicados localizavam-se nos músculos esqueléticos; havia áreas pálidas irregulares nos músculos dos ovinos 5 e 11-13. Essas alterações eram discretas nos músculos dos ovinos 5 e 12 e moderadas nos dos ovinos 11 e 13 . Foram observadas nos músculos masseter, semitendíneo (Fig. 4), extrínsecos da língua nos ovinos 5,11 e 13, esternocefálicos, braquicefálicos e peitorais (ovinos 5 e 11), supraespinal (5 e 13), redondo maior, esofágico (11), abdominais, vasto lateral, vasto medial, vasto intermédio e reto da coxa (13), e músculos do pescoço (12). Em um ovino (11) havia edema periesofágico e ventral aos músculos esternocefálico e braquicefálico. Os ovinos 2 ,
12, 13 e 14 apresentaram aumento da quantidade (de 50 a $75 \mathrm{ml}$ ) de líquido citrino no saco pericárdico.

Três ovinos apresentaram lesões macroscópicas no miocárdio, caracterizadas por discretas áreas pálidas focais e irregulares (ovinos 5 e 12), mais concentradas no miocárdio do ventrículo direito no ovino 12 ; ou por palidez difusa discreta no ventrículo direito do ovino 13 . Edema pulmonar foi observado em quatro ovinos (2, 3,12 e 14). Era discreto nos ovinos 3 e 12 e acentuado nos ovinos 2 e 14 . 0 ovino 2 apresentou congestão pulmonar acentuada. O conteúdo do intestino delgado era semi-líquido e vermelho-achocolatado em quatro ovinos (3 e 12-14), continha ocasionais coágulos sanguíneos em um (ovino 3), e estava associado a avermelhamento da mucosa dos segmentos iniciais do intestino delgado (ovinos 3, 12, 13 e 14). O fígado estava moderadamente pálido em três ovinos ( 3,5 e 13) e de forma discreta em um (ovino 12). No ovino 3 havia edema moderado da parede da vesícula biliar, principalmente nas áreas em contato com o fígado. A urina dos ovinos 5 e 11 era escura, acastanhada. Os rins do ovino 14 estavam vermelho-escuros. Em um animal (ovino 2) os linfonodos pré-escapulares estavam avermelhados e suculentos ao corte. Na cavidade oral do ovino 13 havia uma massa de alimento compactado e pouco mastigado. Nos ovinos 1 e 4 e no ovino controle (10) não foram encontradas lesões macroscópicas.

\section{Achados histopatológicos}

As lesões histológicas dos músculos esqueléticos e da língua caracterizavam-se por tumefação, ausência de estriações longitudinais e transversais e eosinofilia multifocais, eventualmente coalescentes, de segmentos de miofibras. Nos cortes transversais observava-se perda da forma poligonal característica das miofibras, que ficavam arredondadas, tumefeitas e com marcada eosinofilia. Essas alterações caracterizavam necrose hialina segmentar multifocal (Fig. 5). Ocorria fragmentação de material sarcoplasmático com formação de agregados irregulares de material eosinofílico contidos dentro da membrana basal (necrose flocular). Degeneração vacuolar foi também observada.

Infiltrado inflamatório composto predominantemente por macrófagos, muitas vezes fagocitando fragmentos necróticos de miofibras, céliulas satélites e raros linfócitos e neutrófilos, ocorria freqüentemente associado às áreas com degeneração hialina e flocular. Nos segmentos musculares afetados e porções adjacentes havia mobilização das células satélites, que apareciam aumentadas de volume, tinham núcleos vesiculares, e, nos casos em que havia necrose flocular, localizavam-se no interior das miofibras (Fig. 5).

Nos músculos esqueléticos de dois animais (ovinos $4 \mathrm{e}$ 13), que morreram respectivamente 4 e 9 dias após a administração de narasina, e na língua do ovino 13 , foram observadas lesões regenerativas. Constituem-se de fibras delgadas, moderadamente basofílicas, com ausência de estriações e com núcleos grandes, dispostos em fileiras nas porções mais centrais dos filamentos (formação de miotubos).

Quanto a frequência e intensidade das lesões observadas 
nas seções de músculos esquelético havia, em ordem decrescente, necrose flocular, necrose hialina, presença de macrófagos e eventos de fagocitose de miofibras lesadas, ativação de células satélites, e muito menos intensamente, formação de miotubos e degeneração vacuolar. Os músculos esqueléticos mais afetados foram, em ordem de intensidade e frequência, língua, reto da coxa, semitendíneo, abdominal, tríceps, supra-espinhal, vasto medial, vasto lateral e intermédio, diafragma e longissimus dorsi, respectivamente. 0 músculo ocular não estava afetado em nenhum dos casos. Lesões musculares na língua estavam presentes em seis animais (ovinos 4,5 e 11-14), sendo mais acentuadas nos ovinos 5,11 e 13.

O ovino 14 só apresentou lesões discretas na língua e o ovino 10 (controle) não apresentou lesões.

Alterações histológicas no miocárdio eram mais discretas que as observadas nos músculos esqueléticos. Consistiam principalmente de necrose hialina e infiltrado de macrófagos fagocitando restos de miofibras e de linfócitos. Necrose flocular e degeneração vacuolar ocorriam com menor intensidade. Focos discretos de perda de miofibras e fibrose passiva foram observados em algumas seções de músculo cardíaco dos ovinos 1,5,11 e 13. Fibroplasia cardíaca foi observada em um ovino que sobreviveu por 9 dias após a administração de narasina. Não ocorreram alterações histológicas no miocárdio dis ovinos 2,3,10 e 14 .

Vacuolização do citoplasma de hepatócitos foi observada no fígado de seis ovinos $(1,4,5,12,13$ e 14). Essa era difusa em três casos (ovinos 12, 13 e 14), sendo acentuada no ovino 13 e moderada nos ovinos 12 e 14 . O fígado do ovino 5 apresentava vacuolização moderada dos hepatócitos periportais. Nos ovinos 1 e 4 , a vacuolização era centrolobular, moderada e discreta, respectivamente.

\section{DISCUSSÃO}

Narasina, em administrações orais, mostrou-se tóxica para ovinos. O quadro clínico apresentado pelos ovinos intoxicados por narasina consistiu basicamente de hipertermia, distúrbios respiratórios, gastrintestinais e distúrbios relacionados a incapacitação muscular.

A elevação da temperatura, constatada em cinco ovinos (38\% dos animais que receberam a droga), teve início 2 a 7 horas após a administração de narasina, e diminuiu até desaparecer em cerca de 7 a 10 horas. Esta manifestação não é relatada em qualquer espécie animal intoxicada por antibióticos ionóforos e foi considerada importante neste estudo, pela frequência de seu aparecimento. A causa do aumento de temperatura nos ovinos desse experimento não é evidente. Essa alteração clínica não foi associada nem proporcional ao grau de lesão muscular. Embora dois ovinos que apresentaram febre tivessem lesões musculares acentuadas e mioglobinúria, outros dois apresentaram apenas lesões discretas e, em um ovino, não havia lesão macroscópica ou microscópica. Uma possibilidade é que a elevação de temperatura tenha resultado da ação direta da droga, ou de algum metabólito gerado por ela, agindo como pirogênio exógeno.
Os distúrbios respiratórios consistiram de respiração entrecortada, ofegante e taquipnéia, e tiveram início em torno de 2 a 24 horas após a ingestão de narasina. Esses sinais atenuaram e desapareceram em até 30 horas, mesmo nos animais que morreram. Sinais clínicos semelhantes são relatados em ovinos (Newsholme et al. 1983, Fourie et al. 1991), bovinos (Collins \& McCrea 1978, Geor \& Robinson 1985, Fourie et al. 1991), suínos (Van Halderen et al. 1993) e cães (Karsai et al. 1990). Em animais intoxicados com antibióticos ionóforos que apresentaram edema pulmonar associado aos sinais de distúrbios respiratórios, a lesão pulmonar tem sido responsabilizada por esses sinais clínicos. Em dois dos quatro ovinos desse estudo com edema pulmonar essa alteração era acentuada. Alternativamente ou concomitantemente é possível que os sinais clínicos sejam devidos a incapacitação funcional dos músculos envolvidos na respiração, associada ou não a alterações morfológicas.

Dentre os sinais clínicos de distúrbios gastrintestinais, a perda total ou parcial do apetite foi frequente. Em menor número de ovinos houve atonia ruminal, timpanismo e alteração na consistência das fezes. Diarréia tem sido associada à intoxicação por antibióticos ionóforos em ovinos (Nation et al. 1982, Confer et al. 1983, Anderson et al. 1984), bovinos (Van Vleet et al. 1983, Geor \& Robinson, 1985), mas a patogenia é incerta. A anorexia é um dos sinais clínicos mais constantes na intoxicação por antibióticos ionóforos (Novilla 1992). Tem sido observada nessa intoxicação em ovinos (Anderson et al. 1984, Bourque et al.1986, Fourie et al.1991), bovinos (Collins \& McCrea 1978, Van Vleet et al. 1983, Van Vleet \& Ferrans 1983, Shlosberg et al. 1986,1992), suínos (Van Halderen et al. 1993), caninos (Wilson 1980, Karsai et al. 1990), equinos (Rollinson et al. 1987), coelhos (Salles et al. 1994). É sugerido que a perda parcial ou total do apetite, comuns na intoxicação por antibióticos ionóforos, sejam devidas a alteração na palatabilidade da ração, causada pela presença do antibiótico ionóforo e que funcionaria como um fator de defesa do animal, evitando a ingestão de quantidades maiores da toxina (Shlosberg et al. 1986). Neste experimento, no entanto, em que a administração da droga foi dissociada da ração, esses sinais clínicos devem ser devidos a alterações fisiopatológicas induzidas pela droga.

Os distúrbios relacionados à incapacitação muscular observados nos ovinos intoxicados abrangeram principalmente alterações na locomoção e na apreensão de alimentos. Sinais semelhantes têm sido descritos na intoxicação por narasina e outros antibióticos ionóforos em ovinos (Newsholme et al. 1983, Bourque et al. 1986, Fourie et al. 1991), bovinos (Geor \& Robinson 1985, Fourie et al. 1991), suínos (Van Halderen et al. 1993, Miskimins \& Neiger 1996), coelhos (Salles et al. 1994) e cães (Wilson et al.1980, Karsai et al. 1990). Sinais clínicos de incapacitação muscular sem lesão detectável macroscopicamente ou na microscopia óptica têm sido relatadas em associação à intoxicação por antibióticos ionóforos em ovinos (Confer et al. 1983, Gava, 1991).

Dois ovinos apresentaram mioglobinúria. Essa alteração tem sido descrita associada à intoxicação por antibióticos 
ionóforos em ovinos (Newsholme et al. 1983), bovinos (Van Vleet et al. 1983, Wouters et al. 1997), suínos (Miskimins \& Neiger 1996) e cães (Wilson et al. 1980, Karsai et al. 1990). A presença de mioglobina na urina é indicativa de dano muscular grave (Hulland 1993).

Os animais que receberam as doses mais elevadas $(30 \mathrm{mg} /$ $\mathrm{kg}$ em administração única ou $15 \mathrm{mg} / \mathrm{kg}$ divididos em duas administrações diárias) apresentaram curso clínico agudo, com morte em até 46 horas após administração de narasina. $\mathrm{Na}$ necropsia desses ovinos não se observaram lesões macroscópicas e, em um caso, lesões microscópicas também estavam ausentes. Esse fator deve ser levado em conta no diagnóstico de surtos espontâneos de intoxicação por antibiótico ionóforo em ovinos. Nos ovinos com curso clínico mais longo, as lesões eram mais pronunciadas e havia alterações reparativas acentuadas. A menor dose que causou a morte de ovinos neste experimento foi de $4 \mathrm{mg} / \mathrm{kg}$. Doses totais de $12 \mathrm{mg} / \mathrm{kg}$, fracionadas em 3 doses diárias de $4 \mathrm{mg} / \mathrm{kg}$, causaram a morte de dois ovinos. Doses totais semelhantes (14 $\mathrm{mg}$ ) fracionadas em 14 administrações diárias de $1 \mathrm{mg} / \mathrm{kg}$ provocaram sinais clínicos, porém não causaram a morte.

Um ovino apresentou morte súbita, três dias após a ingestão de narasina. Não foram, no entanto, detectadas lesões no miocárdio. A morte poderia estar relacionada a alterações funcionais ou bioquímicas, sem a presença de alterações morfológicas correspondentes no miocárdio (Newsholme 1982), como já foi relatado em casos de intoxicação por antibióticos ionóforos em coelhos (Salles et al. 1994).

Lesões degenerativas foram observadas na musculatura esquelética em ovinos que morreram a partir de 30 horas após a administração da droga, mas no miocárdio foram observadas apenas em ovinos com curso clínico de, no mínimo, 2 dias.

As lesões foram mais acentuadas nos músculos esqueléticos que no miocárdio, principalmente nos músculos da língua, membros posterior e anterior, e abdominal. Achados semelhantes foram observados na intoxicação experimental e espontânea por antibióticos ionóforos em ovinos (Nation et al. 1982, Newsholme et al. 1983, Bastianello et al. 1995). Seis dos nove ovinos intoxicados que morreram tinham lesões nos músculos da língua. Isso não tem sido descrito na intoxicação por antibióticos ionóforos nessa espécie. No entanto, é mencionada na toxicose em bovinos (Van Vleet et al. 1983). O conhecimento da localização das lesões pode ser útil na colheita de material para diagnóstico histopatológico em casos suspeitos de intoxicação por antibiótico ionóforos em ovinos.

Mineralização de miofibras não foi observada. A destruição precoce das mitocôndrias parede ser responsável pelos baixos níveis de calcificação de segmentos musculares lesados na intoxicação por antibióticos ionóforos (Hulland, 1993). Congestão da mucosa intestinal foi observada em quatro ovinos. Essa mesma alteração foi observada em ovinos (Nation et al.1982, Newsholme et al.1983, ), bovinos (Collins \& McCrea, 1978, Shlosberg et al.1992, Bastianello et al.1995) intoxicados por antibióticos ionóforos e, ocasionalmente interpretada como gastroenterite difusa (Collins \& McCrea, 1978). Nos casos deste estudo não foram encontradas, ao exame microscópico, alterações que justificassem um diagnóstico de enterite.

Os resultados deste experimento, quando comparados com os de experimento semelhante com narasina realizado em bovinos (Wouters et al. 1997) indicam que os ovinos são mais sensíveis que bovinos aos efeitos tóxicos de droga. Doses a partir de $4 \mathrm{mg} / \mathrm{kg}$ foram letais para ovinos, enquanto que em bovinos, a morte ocorreu somente em animais que ingeriram doses iguais ou superiores a $15 \mathrm{mg} / \mathrm{kg}$. A administração de doses inferiores provocou apenas doença clínica discreta, com posterior recuperação. Tal relação já foi encontrada para outros antibióticos ionóforos (Confer et al. 1983).

\section{REFERÊNCIAS}

Anderson T.D., Van Alstine W.G., Ficken M.D., Miskimins D.W., Carson T.L. \& Osweiller G.D. 1984. Acute monensin toxicosis in sheep: Light and electron microscopic changes. Am. J. Vet. Res. 45:1142-1143.

Bastianello S.S., Fourie N., Prozesky L., Nel P.W. \& Kellermann T.S. 1995. Cardiomyopathy of ruminants induced by the litter of poultry fed on rations containing the ionophore antibiotic, maduramicin II. Macropathology and histopathology. Onderstepoort J. Vet. Res. 62:5-18.

Behr K.-P., Lüders H., Glünder G. \& Friedrichs M. 1988. Narasin-Intoxikation bei Puten. Dtsch. Tierärztl. Wschr. 95:107-111.

Bourque J.G., Smart M. \& Wobeser G. 1986. Monensin toxicity in lambs. Can. Vet. J. 27:397-399.

Collins E.A. \& McCrea C.T. 1978. Monensin sodium toxicity in cattle. Vet. Rec. 103:385.

Confer A.W., Reavis D.U. \& Panciera R.J. 1983. Light and electron microscopic changes in cardiac and skeletal muscle of sheep with experimental monensin toxicosis. Vet. Pathol. 20:590-602.

Fourie N., Bastianello S.S., Prozesky L., Nel P.W. \& Kellerman T.S. 1991. Cardiomyopathy of ruminants induced by litter of poultry fed on rations containing the ionophore antibiotic maduromicin. I. Epidemiology, clinical signs and clinical pathology. Onderstepoort J. Vet. Res. 58:291-296.

Gava A. 1991. Comunicação pessoal (UDESC, CAV, Lages, Santa Catarina).

Geor R. J. \& Robinson W.F. 1985. Suspected monensin toxicosis in feedlot cattle. Aust. Vet. J. 62:130-131.

Hulland T.J. 1993. Muscle and tendon, p. 183-265. In: Jubb K.V.F., Kennedy P.C., Palmer N. (ed.) Pathology of Domestic Animals. Vol. 1. 4th ed. Academic Press, San Diego. 780 p.

Karsai F., Papp L., Sályi G., Bagó Gy. \& Kántás K. 1990. Gehäuft auftretende Narazin-Vergiftung bei Hunden. Tierärztl. Umschau 45:316-324.

Miskimins D.W. \& Neiger R.D. 1996. Monensin toxicosis in swine. J. Vet. Diagn. Invest. 8:396-397.

Nation P.N., Crowe S.P. \& Harries W.N. 1982. Clinical signs and pathology of accidental monensin poisoning in sheep. Can. Vet. J. 23: 323-326.

Newsholme S.J. 1982. Reactions patterns in myocardium in response to injury. J. South Afr. Vet. Assoc. 53:52-59.

Newsholme S.J., Howerth E.W., Bastianello S.S., Prozesky L. \& Minné J.A. 1983. Fatal cardiomyopathy in feedlot sheep attributed to monensin toxicosis. J. South Afr. Vet. Assoc. 54:29-32.

Novilla M.N. 1992. The veterinary importance of the toxic syndrome induced by ionophores. Vet. Hum. Toxicol. 34:66-70.

Rollinson J., Taylor F.G.R. \& Chesney J. 1987. Salinomycin poisoning in horses - an international incident. Vet. Rec. 121:126-128.

Salles M.S, Barros C.S.L. \& Barros S.S. 1994. Ionophore antibiotic (narasin) poisoning in rabbits. Vet. Hum. Toxicol. 36:437-444.

Shlosberg A., Perl S., Yakobson B., Klopfer U., Egyed M.N., Nobel T.A. \& Efron Y. 1986. The chronic course of a probable monensin toxicosis in cattle. Vet. Hum. Toxicol. 28:230-233.

Shlosberg A., Harmelin A., Perl S., Pano G., Davidson M., Orgad U., Kali U., Bor A., Van Ham M., Hoida G., Yacobson B., Avidar Y., Israeli B-A. \& Bogin E. 1992. Cardiomyopathy in cattle induced by residues of the coccidiostatic 
maduramicin in poultry litter given as a feedstuff. Vet. Res. Comm. 16(1):45-58.

Van Halderen A., Bastianello S.S., Fourie N. \& Zumpt I.F. 1993. An outbreak of narasin poisoning in swine. J. South Afr. Vet. Assoc. 64(1):43-46.

Van Vleet J.F., Amstutz H.E., Weirich W.E., Rebar A.H. \& Ferrans V.J. 1983. Clinical, clinicopathologic, and pathologic alterations in acute monensin toxicosis in cattle. Am. J. Vet. Res. 44:2133-2144.
Van Vleet J.F. \& Ferrans V.J. 1983 Ultrastructural myocardial alterations in monensin toxicosis of cattle. Am. J. Vet. Res. 44:1629-1636.

Wilson J.S. 1980. Toxic myopathy in a dog associated with the presence of monensin in dry food. Can. Vet. J. 21:30-31.

Wouters A.T.B., Wouters F. \& Barros C.S.L. 1997. Intoxicação experimental por narasina em bovinos. Pesq. Vet. Bras. 17(2):82-88. 